\title{
Controllability of some coupled parabolic systems by one control force
}

\author{
Manuel GONZÁLEZ-BURGOSa ${ }^{\mathrm{a}}$, Rosario PÉREZ-GARCÍA ${ }^{\mathrm{a}}$
}

a Dpto. EDAN, Univ. Sevilla, Aptdo. 1160, 41080 Sevilla, Spain. Phone: (34) 954557999. Fax: (34) 954552898. E-mails: manoloburgos@us.es, rosariopg@us.es

(Reçu le jour mois année, accepté après révision le jour mois année)

Abstract. In this Note we present a new approach which allows one to prove new controllability results for some coupled parabolic systems considered in a bounded domain $\Omega$ of $\mathbb{R}^{N}$ when one controls by a unique distributed control. We analyze, as a model example, the null controllability of a linear phase field system. First, one controls the system by two controls. Then, one eliminates the introduced fictitious control. Global Carleman estimates and the parabolic regularity are used. (c) 2004 Académie des sciences/Éditions scientifiques et médicales Elsevier SAS

Contrôlabilité de quelques systèmes paraboliques couplés avec un seul contrôle.

Résumé. Dans cette Note, on présente une nouvelle approche qui permet de prouver de nouveaux résultats de contrôlabilité pour quelques systèmes paraboliques couplés considerés dans un domaine borné $\Omega$ de $\mathbf{R}^{N}$ et contrôlés par un seul contrôle distribué. On analyse, comme exemple modèle, la contrôlabilité nulle d'un système linéaire de champ de phases. D'abord, on contrôle le système par deux contrôles. Ensuite, on élimine le contrôle artificiel introduit. Des estimations globales de Carleman et la régularité parabolique sont employées. (c) 2004 Académie des sciences/Éditions scientifiques et médicales Elsevier SAS

\section{Version française abrégée}

Soit $\Omega \subset \mathbb{R}^{N}$ un ouvert borné et régulier $(N \geq 1$ arbitraire). Soit $\omega \subset \Omega$ un ouvert non vide arbitrairement petit. Pour $T>0$, on pose $Q=\Omega \times(0, T)$ et $\Sigma=\partial \Omega \times(0, T)$. Comme exemple modèle, on analysera le problème linéaire de contrôlabilité nulle (1)-(2), où $a, c, e \in L^{\infty}(Q)$, $B, F \in L^{\infty}(Q)^{N}, u_{0}, \phi_{0}$ sont donnés dans un espace convenable et $v \in L^{2}(Q)$ est un contrôle à déterminer (on dénote par $\mathbf{1}_{\omega}$ la fonction caractéristique de $\omega$ ). Cette analyse est essentiellement orientée à l'étude de la contrôlabilité nulle du système (12) avec des terms superlinéaires (ce qui généralise le système de champ de phases introduit par Caginalp, cf. [3]). Pour pouvoir appliquer

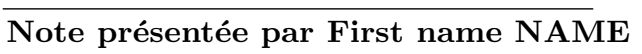

S0764-4442(00)0????-?/FLA

(C) 2004 Académie des sciences/Éditions scientifiques et médicales Elsevier SAS. Tous droits réservés. 1 


\section{González-Burgos, R. Pérez-García}

un argument de point fixe approprié, nous sommes intéressés à obtenir un 'bon' contrôle $v$ au sens que la solution $(u, \phi)$ de $(1)$ associée à $v$ satisfasse $(2)$ et $(u, \phi) \in L^{\infty}(Q)^{2}$. Ce problème est plus complexe que le problème de contrôlabilité nulle pour une équation scalaire de la chaleur linéaire parce qu'il y a des difficultés techniques additionnelles qui viennent du couplage entre les équations.

Dans des travaux récents, ce genre de problèmes a été analysé. Dans [2], les auteurs prouvent la contrôlabilité nulle, avec un seul contrôle, de deux équations de la chaleur en cascade, la première étant de type superlinéaire. Avec une méthode différente, dans [1] les auteurs prouvent la contrôlabilité nulle du système (1) quand $a \equiv e \equiv 0, B \equiv F \equiv 0$ et $N \leq 5$. Dans cette Note, nous étendons ce travail à des systèmes plus généraux. Notre approche est complètement différente de celle utilisée dans [1] et permet d'obtenir des résultats pour $N \geq 1$ arbitraire. Les idées principales de notre stratégie sont les suivantes.

Soient $\mathcal{B}_{0}$ et $\mathcal{B}$ deux ouverts tels que $\mathcal{B}_{0} \subset \subset \mathcal{B} \subset \omega$. Dans un premier temps, on introduit un contrôle artificiel dans (1) et on construit (voir la Proposition 2.1) deux contrôles $\hat{v}_{1}, \hat{v}_{2} \in L^{2}(Q)$, à support dans $\overline{\mathcal{B}}_{0} \times[0, T]$, qui donnent la contrôlabilité nulle du système $(3)$, grâce à l'inégalité d'observabilité (5) pour les solutions du système adjoint.

Dans une deuxième étape, on élimine $\hat{v}_{2}$ et on construit un contrôle $v \in L^{r}(Q)(r \in[2, \infty))$ tel que la solution $(u, \phi)$ de $(1)$ associée à $v$ appartienne à $L^{\infty}(Q)^{2}$ et satisfasse $(2)$. D'abord, on considère une fonction $\eta \in C^{\infty}([0, T])$ telle que $\eta \equiv 1$ dans $[0, T / 3], \eta \equiv 0$ dans $[2 T / 3, T]$ et $0 \leq \eta \leq 1$, $\left|\eta^{\prime}(t)\right| \leq C / T$ dans $[0, T]$ et on introduit le changement des variables $U=u-\eta \bar{u}, \Phi=\phi-\eta \bar{\phi}$, où $(\bar{u}, \bar{\phi})$ résout $(3)$ avec $v_{1} \equiv v_{2} \equiv 0$. Il est clair qu'un contrôle $v$ résout (1)-(2) si et seulement si $v$ résout le problème de contrôlabilité nulle (6). Ainsi, il suffit d'obtenir un contrôle dans $L^{r}(Q)$ qui résout $(6)$. À cet effet, on note $(\hat{u}, \hat{\phi})$ la solution de $(3)$ associée aux contrôles $\hat{v}_{1}$ et $\hat{v}_{2}$. On peut écrire que $\hat{u}=\hat{U}+\eta \bar{u}$ et $\hat{\phi}=\hat{\Phi}+\eta \bar{\phi}$, où $\eta$ et $(\bar{u}, \bar{\phi})$ sont comme ci-dessus et $(\hat{U}, \hat{\Phi})$ est la solution de (7). Ensuit, soit $\theta \in \mathcal{D}(\mathcal{B})$ telle que $\theta \equiv 1$ dans un voisinage de $\mathcal{B}_{0}$. On pose alors

$$
\begin{gathered}
\Phi=(1-\theta) \hat{\Phi}, \quad U=(1-\theta) \hat{U}+\theta \eta^{\prime} \bar{\phi}+2 \nabla \theta \cdot \nabla \hat{\Phi}+(\Delta \theta) \hat{\Phi}, \\
v=\quad \theta \eta^{\prime} \bar{u}-2 \nabla \theta \cdot \nabla \hat{\Phi}-(\Delta \theta) \hat{\Phi}+2 \nabla \theta \cdot \nabla \hat{U}+(\Delta \theta) \hat{U}-\nabla \theta \cdot(B \hat{U})-\nabla \theta \cdot(F \hat{\Phi}) \\
+\quad\left(\partial_{t}-\Delta+B \cdot \nabla+a\right)\left[\theta \eta^{\prime} \bar{\phi}+2 \nabla \theta \cdot \nabla \hat{\Phi}+(\Delta \theta) \hat{\Phi}\right] .
\end{gathered}
$$

En utilisant les propriétés de régularisation parabolique locale de $(\bar{u}, \bar{\phi})$ et $(\hat{U}, \hat{\Phi})$, avec des hypothèses appropriées sur les données initiales et sur le potentiel $c$, on montre que les fonctions $u=U+\eta \bar{u}, \phi=\Phi+\eta \bar{\phi}$ et le contrôle $v$ sont assez réguliers et on a des estimations convenables pour $(u, \phi)$ et $v$ (voir $(10)$ et $(11))$. En outre, $v$ et $(u, \phi)$ résolvent $(6)$, donc $v$ et $(u, \phi)$ résolvent (1)-(2). Plus précisement, on a le Théorème 3.1. Les détails sont donnés dans [5].

On finit cette Note avec d'autres résultats et remarques.

\section{Introduction}

The main objective of this Note is to present a new approach which allows one to prove new controllability results for some (linear or nonlinear) coupled parabolic systems considered in a bounded domain when one controls by a unique distributed control.

Let $\Omega \subset \mathbb{R}^{N}$ be a bounded domain with boundary $\partial \Omega$ of class $C^{2}$ (with $N \geq 1$ being arbitrary). Let $\omega \subset \Omega$ be an arbitrary nonempty open set. For $T>0$, we denote $Q=\Omega \times(0, T)$ and $\Sigma=\partial \Omega \times(0, T)$. In order to describe our strategy, we will concentrate on analyzing, as a model example, the linear null controllability problem:

$$
\left\{\begin{array}{l}
\partial_{t} u-\Delta u+B \cdot \nabla u+a u+F \cdot \nabla \phi+e \phi=-\Delta \phi+v \mathbf{1}_{\omega} \quad \text { in } Q \\
\partial_{t} \phi-\Delta \phi+c \phi=u \quad \text { in } Q \\
u=0, \quad \phi=0 \quad \text { on } \Sigma, \quad u(x, 0)=u_{0}(x), \quad \phi(x, 0)=\phi_{0}(x) \quad \text { in } \Omega
\end{array}\right.
$$




$$
u(x, T)=0, \quad \phi(x, T)=0 \quad \text { in } \Omega,
$$

where $a, c, e \in L^{\infty}(Q), B, F \in L^{\infty}(Q)^{N}, u_{0}, \phi_{0} \in L^{2}(\Omega)$ (at least), and $v \in L^{2}(Q)$ is a control function to be determined (here, $\mathbf{1}_{\omega}$ denotes the characteristic function of $\omega$ ). System (1) is a linearized version of the phase field model introduced by Caginalp (cf. [3]), which provides a mathematical description of the physical phenomenon of solidification of a liquid (the function $u=u(x, t)$ represents the enthalpy of the system and $\phi=\phi(x, t)$ is the so-called phase field function).

As is well-known (see [4] and [8], for instance), the null controllability of a similar nonlinear coupled system would be obtained by combining a null controllability result for (1) and an appropriate fixed-point argument. The present study is mainly directed towards the analysis of the interesting case when superlinear nonlinearities (with a moderate growth at infinity) are considered. Thus, we are interested in obtaining a 'good' control $v$ to the effect that the corresponding solution $(u, \phi)$ to (1) not only satisfies (2) but also lies in $L^{\infty}(Q)^{2}$. Furthermore, appropriate estimates of the control $v$ and the solution $(u, \phi)$ with respect to the size of the data must be obtained. This problem is more intricate than the null controllability problem for a scalar heat equation since, even being a linear problem, additional technical difficulties arise owing to the coupling of the equations.

In recent papers, the controllability properties of superlinear coupled parabolic systems by one control force have been analyzed. In [2], the authors introduce a new technique to construct regular controls from $L^{2}$-controls and prove the null controllability of a cascade system of two heat equations where the second one is controlled by a control function which acts on it indirectly through the solution of the first one. In [1], the authors consider system (1) with $a \equiv e \equiv 0$ and $B \equiv F \equiv 0$. By using a different approach, they prove a 'refined' observability inequality (for the adjoint system) which allows them to obtain controls in $L^{q_{N}}(Q)$ (together with appropriate estimates) that give the null controllability of the system, with $q_{N} \in(2, \infty)$ if $N=1,2$ and $N / 2+1<q_{N} \leq 2(N+2) /(N-2)$ if $3 \leq N<6$. A suitable fixed-point reformulation gives a similar result in the nonlinear case.

In the present Note we extend the results in [1]. It is interesting to remark that our approach is completely different from the one used in [1] and makes it possible to obtain controllability results which are valid for arbitrary dimensions $N \geq 1$. A brief idea of our strategy is as follows. First, we introduce a fictitious control in the second PDE of (1) and prove the null controllability of the system with two controls $\hat{v}_{1}$ and $\hat{v}_{2}$ in $L^{2}(Q)$. In a second step, we eliminate $\hat{v}_{2}$ and construct a 'good' control $v$ that solves (1)-(2). In the next two Sections, we just sketch these main steps and the details will be given in [5]. We end this Note with further results and comments.

\section{The linear null controllability problem with two controls}

Let $\mathcal{B}_{0}$ be a nonempty open set such that $\mathcal{B}_{0} \subset \subset \omega$. Let us consider the linear system

$$
\left\{\begin{array}{l}
\partial_{t} u-\Delta u+B \cdot \nabla u+a u+F \cdot \nabla \phi+e \phi=-\Delta \phi+v_{1} \mathbf{1}_{\mathcal{B}_{0}} \quad \text { in } Q, \\
\partial_{t} \phi-\Delta \phi+c \phi=u+v_{2} \mathbf{1}_{\mathcal{B}_{0}} \quad \text { in } Q, \\
u=0, \phi=0 \quad \text { on } \Sigma, \quad u(x, 0)=u_{0}(x), \quad \phi(x, 0)=\phi_{0}(x) \quad \text { in } \Omega .
\end{array}\right.
$$

The following null controllability result holds:

Proposition 2.1. - Let $a, c, e \in L^{\infty}(Q), B, F \in L^{\infty}(Q)^{N}$, and $u_{0}, \phi_{0} \in L^{2}(\Omega)$ be given. Then, there exist two control functions $\hat{v}_{1}, \hat{v}_{2} \in L^{2}(Q)$, with supp $\hat{v}_{1}$, supp $\hat{v}_{2} \subset \overline{\mathcal{B}}_{0} \times[0, T]$, such that the corresponding solution $(\hat{u}, \hat{\phi})$ to $(3)$ satisfies $(2)$. Moreover, $\hat{v}_{1}$ and $\hat{v}_{2}$ can be chosen so that

$$
\left\|\hat{v}_{1}\right\|_{L^{2}(Q)}^{2}+\left\|\hat{v}_{2}\right\|_{L^{2}(Q)}^{2} \leq \exp (C H)\left(\left\|u_{0}\right\|_{L^{2}(\Omega)}^{2}+\left\|\phi_{0}\right\|_{L^{2}(\Omega)}^{2}\right)
$$




\section{González-Burgos, R. Pérez-García}

with $C=C\left(\Omega, \mathcal{B}_{0}\right)>0$ and $H=H(T, a, c, e, B, F)>0$ given by

$$
\begin{aligned}
H & =1+\frac{1}{T}+\|a\|_{\infty}^{2 / 3}+\|c\|_{\infty}^{2 / 3}+\|e\|_{\infty}^{1 / 2}+\|B\|_{\infty}^{2}+\|F\|_{\infty} \\
& +T\left(1+\|a\|_{\infty}+\|c\|_{\infty}+\|e\|_{\infty}+\|B\|_{\infty}^{2}+\|F\|_{\infty}^{2}\right) .
\end{aligned}
$$

The proof of this Proposition is a consequence of the following observability inequality for the solutions to the corresponding adjoint system:

$$
\|\varphi(0)\|_{L^{2}(\Omega)}^{2}+\|\psi(0)\|_{L^{2}(\Omega)}^{2} \leq \exp (C H) \iint_{\mathcal{B}_{0} \times(0, T)}\left(|\varphi|^{2}+|\psi|^{2}\right) d x d t
$$

( $C$ and $H$ as in Proposition 2.1), which is deduced by combining an appropriate Carleman inequality and the energy estimates for the adjoint system (see the details in [5]).

\section{The linear null controllability problem with one control}

We now eliminate $\hat{v}_{2}$ and construct a control $v \in L^{r}(Q)(r \in[2, \infty))$ such that the solution $(u, \phi)$ to $(1)$ lies in $L^{\infty}(Q)^{2}$ and satisfies $(2)$. This can be carried out by adapting the technique introduced in [2].

We proceed as follows. Let $\eta \in C^{\infty}([0, T])$ be such that $\eta \equiv 1$ in $[0, T / 3], \eta \equiv 0$ in $[2 T / 3, T]$, and $0 \leq \eta \leq 1,\left|\eta^{\prime}(t)\right| \leq C / T$ in $[0, T]$. We introduce the change of variables $U=u-\eta \bar{u}, \Phi=\phi-\eta \bar{\phi}$ where $(\bar{u}, \bar{\phi})$ solves $(3)$ with $v_{1} \equiv v_{2} \equiv 0$. It is clear that a control $v$ solves the null controllability problem (1)-(2) if and only if $v$ solves:

$$
\left\{\begin{array}{l}
\partial_{t} U-\Delta U+B \cdot \nabla U+a U+F \cdot \nabla \Phi+e \Phi=-\Delta \Phi-\eta^{\prime} \bar{u}+v \mathbf{1}_{\omega} \text { in } Q, \\
\partial_{t} \Phi-\Delta \Phi+c \Phi=U-\eta^{\prime} \bar{\phi} \text { in } Q \\
U=0, \quad \Phi=0 \quad \text { on } \Sigma, \quad U(x, 0)=0, \quad \Phi(x, 0)=0, \quad U(x, T)=0, \quad \Phi(x, T)=0 \quad \text { in } \Omega .
\end{array}\right.
$$

Thus, it suffices to obtain a control in $L^{r}(Q)$ that solves $(6)$. To this end, let $(\hat{u}, \hat{\phi})$ be the solution to (3) associated to two controls $\hat{v}_{1}$ and $\hat{v}_{2}$ provided by Proposition 2.1. We can also write $\hat{u}=\hat{U}+\eta \bar{u}$ and $\hat{\phi}=\hat{\Phi}+\eta \bar{\phi}$, where $\eta$ and $(\bar{u}, \bar{\phi})$ are as above and $(\hat{U}, \hat{\Phi})$ is the solution to

$$
\left\{\begin{array}{l}
\partial_{t} \hat{U}-\Delta \hat{U}+B \cdot \nabla \hat{U}+a \hat{U}+F \cdot \nabla \hat{\Phi}+e \hat{\Phi}=-\Delta \hat{\Phi}-\eta^{\prime} \bar{u}+\hat{v}_{1} \mathbf{1}_{\mathcal{B}_{0}} \text { in } Q \\
\partial_{t} \hat{\Phi}-\Delta \hat{\Phi}+c \hat{\Phi}=\hat{U}-\eta^{\prime} \bar{\phi}+\hat{v}_{2} \mathbf{1}_{\mathcal{B}_{0}} \text { in } Q \\
\hat{U}=0, \quad \hat{\Phi}=0 \quad \text { on } \Sigma, \quad \hat{U}(x, 0)=0, \quad \hat{\Phi}(x, 0)=0 \quad \text { in } \Omega
\end{array}\right.
$$

which also satisfies $\hat{U}(x, T)=0$ and $\hat{\Phi}(x, T)=0$ in $\Omega$. Now we consider a new open set $\mathcal{B}$ such that $\mathcal{B}_{0} \subset \subset \mathcal{B} \subset \subset \omega$ and a function $\theta \in \mathcal{D}(\mathcal{B})$ satisfying $\theta \equiv 1$ in a neighborhood of $\mathcal{B}_{0}$. We set

$$
\begin{gathered}
\Phi=(1-\theta) \hat{\Phi}, \quad U=(1-\theta) \hat{U}+\theta \eta^{\prime} \bar{\phi}+2 \nabla \theta \cdot \nabla \hat{\Phi}+(\Delta \theta) \hat{\Phi}, \\
v=\quad \theta \eta^{\prime} \bar{u}-2 \nabla \theta \cdot \nabla \hat{\Phi}-(\Delta \theta) \hat{\Phi}+2 \nabla \theta \cdot \nabla \hat{U}+(\Delta \theta) \hat{U}-\nabla \theta \cdot(B \hat{U})-\nabla \theta \cdot(F \hat{\Phi}) \\
+\quad\left(\partial_{t}-\Delta+B \cdot \nabla+a\right)\left[\theta \eta^{\prime} \bar{\phi}+2 \nabla \theta \cdot \nabla \hat{\Phi}+(\Delta \theta) \hat{\Phi}\right] .
\end{gathered}
$$

By the local parabolic regularity of $(\hat{U}, \hat{\Phi})$ and $(\bar{u}, \bar{\phi})$, it is proved that, under appropriate assumptions on the initial data and on the potential $c$, the functions $u=U+\eta \bar{u}, \phi=\Phi+\eta \bar{\phi}$ (with $(U, \Phi)$ defined in (8)) and the above-introduced control $v$ are regular enough, and suitable estimates for $(u, \phi)$ and $v$ hold. In addition, $v$ (together with $(U, \Phi))$ solves $(6)$, thus $v$ (together with $(u, \phi)$ ) solves (1)-(2). To be precise, the following null controllability result is proved: 
TheOREM 3.1. - Let $r, s_{1} \in[2, \infty)$ be given and set

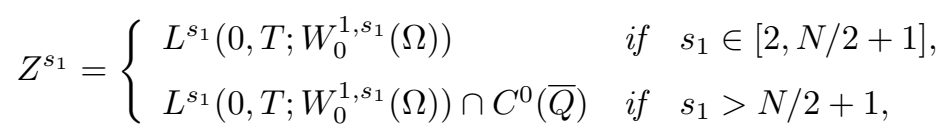

and $X^{s_{1}}=\left\{u: u \in L^{s_{1}}\left(0, T ; W^{2, s_{1}}(\Omega) \cap W_{0}^{1, s_{1}}(\Omega)\right), \partial_{t} u \in L^{s_{1}}(Q)\right\}$. Assume that $u_{0}, \phi_{0} \in$ $W^{2-2 / s_{1}, s_{1}}(\Omega) \cap H_{0}^{1}(\Omega), a, e \in L^{\infty}(Q), B, F \in L^{\infty}(Q)^{N}$, and $c \in L^{\infty}(Q) \cap L^{\gamma}\left(0, T ; W^{1, \gamma}(\Omega)\right)$, with

$$
\gamma= \begin{cases}\max \{r, N / 2+1\} & \text { if } r \neq N / 2+1, \\ N / 2+1+\varepsilon(\varepsilon>0 \text { arbitrarily small }) & \text { if } \quad r=N / 2+1 .\end{cases}
$$

Then, there exists a control function $v \in L^{r}(Q)$, with $\operatorname{supp} v \subset \omega \times[0, T]$, such that the associated solution $(u, \phi)$ to (1) lies in $Z^{s_{1}} \times X^{s_{1}}$ and satisfies (2). Moreover,

$$
\begin{gathered}
\|u\|_{Z^{s_{1}}}+\|\phi\|_{X^{s_{1}}} \leq \exp (C H)\left(\left\|u_{0}\right\|_{W^{2-2 / s_{1}, s_{1}}(\Omega)}+\left\|\phi_{0}\right\|_{W^{2-2 / s_{1}, s_{1}}(\Omega)}\right), \\
\|v\|_{L^{r}(Q)} \leq \exp (C H)\left(1+\|\nabla c\|_{L^{\gamma}(Q)}\right)\left(\left\|u_{0}\right\|_{L^{2}(\Omega)}+\left\|\phi_{0}\right\|_{L^{2}(\Omega)}\right),
\end{gathered}
$$

with $C=C(\Omega, \omega)>0$ and $H=H(T, a, c, e, B, F)>0$ given by (4).

Remark 1. - By following the proof of Theorem 3.1, it is seen that the regularity of $(u, \phi)$ (resp. estimate (10)) is obtained independent of the regularity of the control $v$ (resp. estimate (11)). Indeed, the regularity of $v$ only depends on the local parabolic regularizing effect (thus on the regularity of the term $\nabla c)$, while the regularity of $(u, \phi)$ just depends on the regularity of the initial condition $\left(u_{0}, \phi_{0}\right)$.

\section{Further results and comments}

The nonlinear case Let us consider the nonlinear phase field system

$$
\left\{\begin{array}{l}
\partial_{t} u-\Delta u+f(u, \nabla u, \phi, \nabla \phi)=-\Delta \phi+v \mathbf{1}_{\omega} \quad \text { in } Q, \\
\partial_{t} \phi-\Delta \phi+h(\phi)=u \quad \text { in } Q, \\
u=0, \phi=0 \quad \text { on } \Sigma, \quad u(x, 0)=u_{0}(x), \phi(x, 0)=\phi_{0}(x) \quad \text { in } \Omega .
\end{array}\right.
$$

Assume that $f$ is locally Lipschitz-continuous. Thus, for some $L_{\text {loc }}^{\infty}$ functions $g_{i}$ and $G_{i}, i=1,2$,

$$
f(s, p, \sigma, \pi)=f(0,0,0,0)+g_{1}(s, p, \sigma, \pi) s+G_{1}(s, p, \sigma, \pi) \cdot p+g_{2}(s, p, \sigma, \pi) \sigma+G_{2}(s, p, \sigma, \pi) \cdot \pi
$$

for any $(s, p, \sigma, \pi) \in \mathbb{R} \times \mathbb{R}^{N} \times \mathbb{R} \times \mathbb{R}^{N}$. By combining Theorem 3.1 with an appropriate fixed-point argument, the following null controllability result for system (12) is proved (see [5]):

Theorem 4.1. - Let $f: \mathbb{R} \times \mathbb{R}^{N} \times \mathbb{R} \times \mathbb{R}^{N} \rightarrow \mathbb{R}$ be a locally Lipschitz-continuous function such that $f(0,0,0,0)=0$ and let $h \in C^{1}(\mathbb{R})$ satisfy $h^{\prime \prime} \in L_{\mathrm{loc}}^{\infty}(\mathbb{R})$ and $h(0)=0$. Assume:

i) $\forall R>0 \quad \exists M_{R}>0: \quad\left|g_{1}(s, p, \sigma, \pi)\right|+\left|G_{1}(s, p, \sigma, \pi)\right|+\left|g_{2}(s, p, \sigma, \pi)\right|+\left|G_{2}(s, p, \sigma, \pi)\right| \leq M_{R}$ ii) for every $s, \sigma \in[-R, R]$ and $p, \pi \in \mathbb{R}^{N}$;

$$
\left\{\begin{array}{c}
\lim _{|s|+|\sigma| \rightarrow \infty} \frac{\left|g_{1}(s, p, \sigma, \pi)\right|}{\log ^{3 / 2}(1+|s|+|\sigma|)}=0, \quad \lim _{|s|+|\sigma| \rightarrow \infty} \frac{\left|G_{1}(s, p, \sigma, \pi)\right|}{\log ^{1 / 2}(1+|s|+|\sigma|)}=0, \\
\lim _{|s|+|\sigma| \rightarrow \infty} \frac{\left|g_{2}(s, p, \sigma, \pi)\right|}{\log ^{2}(1+|s|+|\sigma|)}=0, \quad \text { and } \lim _{|s|+|\sigma| \rightarrow \infty} \frac{\left|G_{2}(s, p, \sigma, \pi)\right|}{\log (1+|s|+|\sigma|)}=0 \\
\text { uniformly in } p, \pi \in \mathbb{R}^{N} ; \\
\lim _{|\sigma| \rightarrow \infty} \frac{|h(\sigma)|}{|\sigma| \log ^{3 / 2}(1+|\sigma|)}=0 .
\end{array}\right.
$$




\section{González-Burgos, R. Pérez-García}

Then, if $u_{0}, \phi_{0} \in W^{2-2 / s_{1}, s_{1}}(\Omega) \cap H_{0}^{1}(\Omega)$, with $s_{1} \in(N / 2+1, \infty)$, there exists a control $v \in L^{2}(Q)$ such that (12) has a solution $(u, \phi) \in L^{\infty}(Q)^{2}$ that satisfies $u(x, T)=0$ and $\phi(x, T)=0$ in $\Omega$.

Under no restrictions on the growth at infinity of the nonlinearities $f$ and $h$, a local null controllability result for system (12) is similarly deduced. By replacing hypothesis ii) of Theorem 4.1 by a slightly different one, we can also prove the null controllability to the trajectories and the approximate controllability for system (12). These results, which can be found in [5], generalize those obtained in [1].

An alternative proof of Theorem 3.1 Let $\mathcal{B}_{0}$ and $\mathcal{B}$ be two open sets as in Sections 2 and 3. In [7], the author obtains a Carleman inequality for the solutions $(\varphi, \psi)$ to the adjoint system of (1), by means of which some global terms of $\varphi$ and $\psi$ are bounded only in terms of $\psi$ 'localized' in $\mathcal{B}_{0}$. Such a Carleman inequality leads to the observability inequality

$$
\|\varphi(0)\|_{L^{2}(\Omega)}^{2}+\|\psi(0)\|_{L^{2}(\Omega)}^{2} \leq \exp (C H) \iint_{\mathcal{B}_{0} \times(0, T)}|\psi|^{2} d x d t \quad(C \text { and } H \text { as in Proposition 2.1) }
$$

which allows one to proving the existence of a control $\hat{v} \in L^{2}(Q)$, with $\operatorname{supp} \hat{v} \subset \overline{\mathcal{B}}_{0} \times[0, T]$, that gives the null controllability of system (1), under the hypothesis $c \in L^{\infty}(Q)$. If, in addition, $\nabla c \in L^{\gamma}(Q)^{N}$, with $\gamma$ given by (9), a similar argument to the one used in Section 3 allows one to build a new control $v \in L^{r}(Q)(r \in[2, \infty))$, with $\operatorname{supp} v \subset \overline{\mathcal{B}} \times[0, T]$, such that $v$ solves $(1)-(2)$ and $(u, \phi) \in L^{\infty}(Q)^{2}$.

Null controllability of $m$ coupled parabolic PDEs by one control force By adapting the strategy introduced in this Note, one can control to zero, by a unique distributed control, some cascade systems of $m$ linear coupled parabolic PDEs. First, we would obtain $m$ controls that give the null controllability of the system, in view of an appropriate observability inequality for the adjoint system. Then, we would eliminate the $m-1$ fictitious controls. Only terms of order zero are allowed in the $m-1$ last equations and suitable assumptions on the potentials are required. An appropriate fixed-point argument gives a similar result in the nonlinear case.

Other comments A null controllability result for (12) analogous to Theorem 4.1 (as well as the exact controllability to the trajectories and the approximate controllability under slightly different hypothesis) can be obtained for an unbounded domain $\Omega$ such that $\Omega \backslash \bar{\omega}$ is bounded (cf. [6]).

All along this Note, other type of boundary conditions such as Fourier (or Robin) boundary conditions could have also been considered.

\section{References}

[1] F. Ammar Khodja, A. Benabdallah, C. Dupaix, I. Kostin, Controllability to the trajectories of phasefield models by one control force, SIAM J. Control Optim. 42 (5) (2003) 1661-1680.

[2] O. Bodart, M. González-Burgos, R. Pérez-García, Insensitizing controls for a semilinear heat equation with a superlinear nonlinearity, C.R. Acad. Sci. Paris 335 (8) (2002) 677-682.

[3] C. Caginalp, An analysis of a phase phield model of a free boundary, Arch. Rat. Mech. Anal. 92 (1986) 205-245.

[4] C. Fabre, J.-P. Puel, E. Zuazua, Approximate controllability of the semilinear heat equation, Proc. Roy. Soc. Edinburgh Sect. A 125 (1) (1995) 31-61.

[5] M. González-Burgos, R. Pérez-García, Controllability results for some nonlinear coupled parabolic systems by one control force, in preparation.

[6] M. González-Burgos, R. Pérez-García, Controllability of some nonlinear coupled parabolic systems by one control force in unbounded domains, in preparation.

[7] R. Pérez-García, Thesis, Universidad de Sevilla, Sevilla, Spain, 2004.

[8] E. Zuazua, Exact controllability for the semilinear wave equation, J. Math. Pures Appl. 69 (1) (1990) 1-31. 